\title{
RASGOS DEMOGRAFICOS Y ECONOMICOS DE LA REGION CENTRAL DE ESPAÑA
}

$312: 330$

por

\author{
Ignacio Ballester Ros
}

SUMARIO: I. INTRODUCCION.-II. EL TERRITORIO--III. LA POBLACION.-IV. LA GEOECONOMIA: 1. LA INFRAESTRUCTURA. 2. La agricultura. 3. La silvicultura. 4. La Ganaderfa. 5. La explotactón DE MINAS Y CANTERAS.-V. LA INDUSTRIA.-VI. EL COMERCIO.VII. EL SECTOR DE SERVICIOS.

\section{INTRODUCCION}

Las Cámaras Oficiales de Comercio e Industria de Madrid, Toledo, Ciudad Real, Cuenca, Guadalajara, Albacete, Avila, Segovia y Soria, continuando su línea de colaboración para la realización de estudios y trabajos en común, iniciada en 1969, han publicado una monografía sobre La economía regional en 1973, que constituye la quinta edición de la Memoria-Anuario y contiene un análisis demográfico, económico y social de las nueve Provincias reseñadas, que configuran la Región Central de España.

Es una muestra más del renovado interés por los estudios regionales, cada día más numerosos en nuestra Patria. En trabajos anteriores se ha hecho referencia a diversos trabajos sobre Galicia, Cataluña y Extremadura. Estimamos de indudable interés, desde el punto de vista local, prestar hoy la debida atención a esta monografía.

Con abundante información estadística y concisos comentarios, trata en diez capítulos: del territorio, la población, la geoeconomía, 
con un detenido análisis de la industria, el comercio y los servicios, las finanzas, los precios, el trabajo, la previsión y la acción social, materia más que sobrada para una crónica, por lo que forzosamente limitaremos el comentario a los aspectos de mayor significación.

\section{EL TERRITORIO}

Las nueve Provincias no constituyen una Región natural propiamente dicha, ya que las seis primeras enumeradas pertenecen, con características muy semejantes entre sí, a la Submeseta Sur, y difieren de las de Avila, Segovia y Soria, que se ubican en la Submeseta Norte, separándolas de las primeras el Sistema Central.

En conjunto, su extensión superficial es de $112.505 \mathrm{~km}^{2}$, lo que equivale al 22,3 por 100 del territorio nacional, que se distribuye, por Provincias, en esta forma:

\begin{tabular}{|c|c|c|}
\hline & $\begin{array}{c}\text { Extensión } \\
\text { superficial } \\
\left(\mathrm{km}^{2}\right)\end{array}$ & $\begin{array}{c}\text { Porcentaje de la } \\
\text { superficie total } \\
\text { nacional }\end{array}$ \\
\hline $\begin{array}{llllll}\text { Madrid } & \ldots & \ldots & \ldots & \ldots & \ldots\end{array}$ & 7.995 & 1,6 \\
\hline Toledo ... & 15.368 & 3,0 \\
\hline Ciudad Real $\ldots . . . .$. & 19.749 & 3,9 \\
\hline $\begin{array}{llllll}\text { Cuenca } & \ldots & \ldots & \ldots & \ldots & \ldots \\
\end{array}$ & 17.061 & 3,4 \\
\hline $\begin{array}{cccc}\text { Guadalajara } & \ldots & \ldots & \ldots\end{array}$ & 12.190 & 2,4 \\
\hline Albacete $\ldots \ldots \ldots \ldots \ldots$ & 14.858 & 2,9 \\
\hline $\begin{array}{llllllll}\text { Avila } & \ldots & \ldots & \ldots & \ldots & \ldots & \ldots\end{array}$ & 8.048 & 1,6 \\
\hline $\begin{array}{llllll}\text { Segovia } & \ldots & \ldots & \ldots & \ldots & \ldots\end{array}$ & 6.949 & 1,4 \\
\hline $\begin{array}{lllllll}\text { Soria } & \ldots & \ldots & \ldots & \ldots & \ldots & \ldots\end{array}$ & 10.287 & 2,1 \\
\hline
\end{tabular}

La superficie provincial media es, para esta Región, de 12.500 kilómetros cuadrados; cinco Provincias ofrecen una superficie inferior a la media, las más septentrionales, mientras que las más extensas se hallan situadas al sur de la Región considerada.

En la monografía que comentamos se hace una amplia exposición del aspecto general, situación y límites, orografía, hidrografía, comarcas naturales y divisiones administrativas de cada Provincia, tomada de los Nomenclátores provinciales del Censo de Población de 1970, publicados por el Instituto Nacional de Estadística.

Las nueve Provincias ocupan la parte central de la Península y se hallan situadas en la Meseta, la Región natural, fundamento de nuestra geografía. Las seis primeras, de entre las reseñadas, ocu- 
pan una altiplanicie, de una altura media entre 500 a 600 m., que comprenden, en su mayor parte, los cursos alto y medio de los ríos Tajo y Guadiana, con sus redes de afluentes, si bien en su parte oriental envían también sus aguas al Mediterráneo por medio de las redes fluviales, del Segura, Júcar, Turia, y por el río Mesa, hacia el Ebro. Las otras tres Provincias, cuyas aguas recogen los afluentes del Duero por su izquierda, se sitúan en otra altiplanicie de altura media superior a la anterior. Hacia unas y otras se extienden las estribaciones del Sistema Central.

Entre las comarcas naturales comprendidas en las Provincias sitas al sur de la mencionada cordillera destacan, en primer término, por su extensión, que abarca parte de cuatro Provincias, la Mancha. En la Provincia de Ciudad Real distinguimos, además, por su importancia el Valle de Alcudia y los Campos de Calatrava y Montiel; y en la de Toledo, la Sagra. La Alcarria comprende parte de Cuenca y de Guadalajara. En esta última existen también la Campiña y la Sierra, denominación esta última que se aplica a parte de las Provincias de Cuenca, Albacete y Toledo. En la Provincia de Avila podemos citar las comarcas de La Moraña, Tierra de Arévalo y los valles de Amblés y del Tiétar - la Andalucía de Avila-; y en Segovia hay varias zonas, como la Churrería, con los mejores regadíos; la resinera, la cerealista de secano, la de pastos en las estribaciones de la Sierra y la Sierra propiamente dicha.

Las observaciones meteorológicas del periodo 1931-1960, procedentes del Servicio Meteorológico Nacional, permiten fijar las temperaturas medias anuales de las tres Provincias del valle del Duero entre $10,4^{\circ}$ en Avila y Soria y $11,4^{\circ}$ para Segovia; en la Submeseta Sur oscilan entre $11,7^{\circ}$ para Cuenca y $15^{\circ}$ en Toledo. Las máximas anuales oscilan, de una parte, entre 36,5 para Avila y 38 para Soria, y de otra, entre 38 para Guadalajara y 44,2 en Ciudad Real. Las mínimas anuales son: $-14,8$ en Soria y $-20,4$ en Avila; $-9,2$ en Toledo y $-22,4$ en Albacete. Estas cifras, expresadas todas ellas en grados centígrados, reflejan el clima marcadamente continental de esta Región, con una elevación de temperaturas a medida que bajamos hacia el sur, con la excepción de Albacete, que da la temperatura mínima de la Región.

La precipitación media de esta Región, muy escasamente regada pluvialmente, presenta matices diferenciados; desde los $363 \mathrm{~mm}$. anuales de Avila a los 570,3 de Soria, al norte de la Región, y desde los 351,3 de Albacete a los 568,4 de Cuenca. 


\section{LA POBLACION}

EI Nomenclátor General de España de 1970, al facilitar información sobre el número de Municipios y de entidades singulares de población existentes en la Región y en cada una de sus Provincias, permite conocer las formas predominantes de asentamiento de la población.

La Región tiene 5.980 .576 habitantes de hecho, lo que equivale al 17,7 por 100 de la población total española. El detalle por Provincias es éste:

\begin{tabular}{|c|c|c|c|}
\hline & $\begin{array}{l}\text { Número de } \\
\text { Municipios }\end{array}$ & $\begin{array}{l}\text { Número de } \\
\text { entidades }\end{array}$ & $\begin{array}{l}\text { Población } \\
\text { de hecho }\end{array}$ \\
\hline $\begin{array}{llllll}\text { Madrid } \ldots & \ldots & \ldots & \ldots\end{array}$ & 183 & 314 & 3.792 .561 \\
\hline $\begin{array}{lllll}\text { Toledo } & \ldots & \ldots & \ldots & \ldots\end{array}$ & 205 & 252 & 468.925 \\
\hline Ciudad Real ... ... & 98 & 184 & 507.650 \\
\hline $\begin{array}{lllll}\text { Cuenca } & \ldots & \ldots & \ldots & \ldots\end{array}$ & 280 & 377 & 247.158 \\
\hline $\begin{array}{lll}\text { Guadalajara } & \ldots & \ldots\end{array}$ & 335 & 479 & 147.732 \\
\hline $\begin{array}{llll}\text { Albacete } & \ldots & \ldots & \ldots\end{array}$ & 86 & 369 & 335.026 \\
\hline $\begin{array}{lllllll}\text { Avila } & \ldots & \ldots & \ldots & \ldots & \ldots\end{array}$ & 268 & 436 & 203.798 \\
\hline $\begin{array}{lllll}\text { Segovia } & \ldots & \ldots & \ldots & \ldots\end{array}$ & 234 & 391 & 162.770 \\
\hline $\begin{array}{lllllll}\text { Soria } & \ldots & \ldots & \ldots & \ldots & \ldots\end{array}$ & 219 & 525 & 114.956 \\
\hline
\end{tabular}

La población de la Región se halla concentrada en los Munici. pios pequeños por el número de sus habitantes al norte, hasta la línea del Tajo, y más populosos al sur; pero, en todo caso, con un número reducido de entidades de población, 1,7 por Municipio, es decir, no se llega a dos entidades por Municipio. La población media por Municipio es de 3.134 habitantes. La densidad de población es de 53 habitantes por $\mathrm{km}^{2}$, debido al peso de Madrid, la Capital de la Nación; sin ella, la densidad se reduce a 25 . La población que vive en diseminado equivale solamente al 1,5 por 100 de la población total de la Región.

El detalle por Provincias permitirá un conocimiento más exacto de este fenómeno: 


\begin{tabular}{|c|c|c|c|}
\hline & $\begin{array}{l}\text { Población media } \\
\text { por Municipio }\end{array}$ & $\begin{array}{l}\text { Entidades } \\
\text { por Municipio }\end{array}$ & Densidad \\
\hline Madrid & 20.724 & 1,7 & 474 \\
\hline $\begin{array}{llll}\text { Toledo } & \ldots & \ldots & \ldots\end{array}$ & 2.287 & 1,2 & 31 \\
\hline Ciudad Real... ... & 5.180 & 1,9 & 26 \\
\hline $\begin{array}{lllll}\text { Cuenca } & \ldots & \ldots & \ldots\end{array}$ & 882 & 1,3 & 14 \\
\hline Guadalajara ... ... & 441 & 1,4 & 12 \\
\hline Albacete $\ldots \ldots \ldots$ & 3.895 & 4,3 & 23 \\
\hline $\begin{array}{lllll}\text { Avila } & \ldots & \ldots & \ldots & \ldots\end{array}$ & 760 & 1,6 & 25 \\
\hline $\begin{array}{llll}\text { Segovia } & \ldots & \ldots & \ldots\end{array}$ & 695 & 1,7 & 23 \\
\hline $\begin{array}{llllll}\text { Soria } & \ldots & \ldots & \ldots & \ldots\end{array}$ & 524 & 2,4 & 11 \\
\hline
\end{tabular}

Se advierte en la tabla anterior una elevada población media por Municipio para la Provincia de Madrid, una vez más debido al peso del Municipio de la Capital, pues, sin tener en cuenta a éste, dicho promedio baja a 3.552. Dado el promedio antes fijado para el conjunto de la Región, puede observarse que los Municipios más populosos corresponden a las Provincias de Ciudad Real, Albacete, Madrid y Toledo, al sur de la Región; en las demás es evidente que predomina el Municipio pequeño. También Albacete y Ciudad Real ofrecen una mayor dispersión de la población, con Soria, dado el número de entidades que tienen por Municipio. La densidad de población provincial es significativa; la más alta es la de Madrid, reducida a 81 habitantes por $\mathrm{km}^{2}$ si no tenemos en cuenta a Madrid Capital; en orden de importancia le sigue Toledo; Ciudad Real y Albacete, al sur, y Avila y Segovia, al norte, tienen valores muy similares; las mínimas corresponden a Soria, Guadalajara y Cuenca, a caballo del Sistema Central y de la Cordillera Ibérica. La población diseminada presenta también sus valores más altos en Albacete, Ciudad Real y Todelo, en especial en la primera, con el 19,4 por 100 de la población viviendo fuera de núcleos urbanos, y algo más del 2 las dos restantes.

El incremento de la población en la Región fue muy importante de 1950 a 1960, superando los 600.000 habitantes, y se acrecentó a partir de 1960 debido al rápido crecimiento de Madrid, que aumentó en 1.200.000 habitantes. La evolución más reciente se caracteriza, sin embargo, por una tendencia decreciente en la evolución de la población de todas las Provincias, salvo Madrid, que, si bien aumentó en casi dos millones sus habitantes de 1950 a 1970, ha visto últimamente cómo se reduce su ritmo de incremento. Sin 
embargo, todas las capitales de Provincia han registrado un aumento moderado, mientras que el de Madrid en veinte años fue de 1.600 .000 .

La estructura de la población, refiere el Censo de Población de 1970 , tiene una trascendente importancia económica. Por edades es como sigue, expresada en porcentajes:

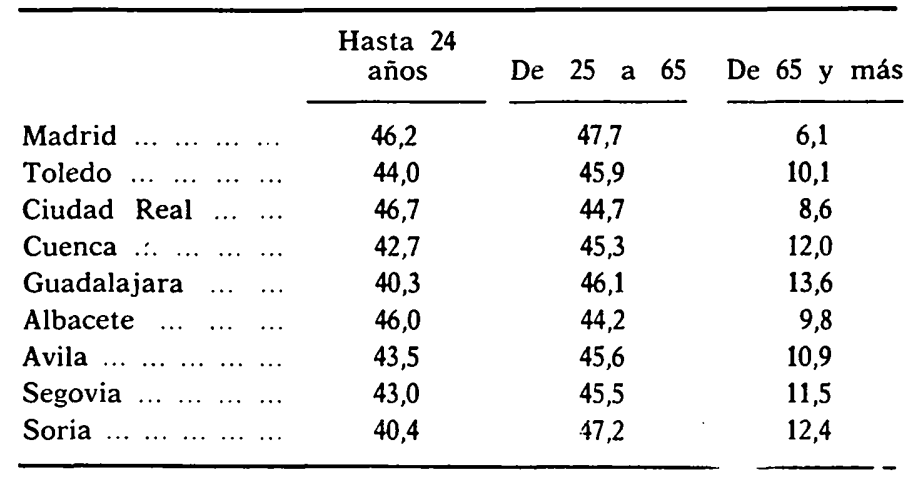

Para el conjunto de la Región, la población de sesenta y cinco y más años representa tan sólo el 7,9 por 100 del total; sólo Madrid ofrece un porcentaje inferior; las demás Provincias se hallan por encima del promedio, en especial las tres Provincias del norte, y al sur, Guadalajara, Cuenca y Toledo. Ello se debe a la emigración. de gentes predominantemente jóvenes, que resulta más acentuada en todas las Provincias, con excepción de Madrid, que, por el contrario, recibe una fuerte inmigración de gentes jóvenes. Sólo Madrid, Ciudad Real y Albacete presentan un porcentaje de personas con edades de hasta veinticuatro años, superior al promedio de la Región.

El crecimiento vegetativo, fruto de la diferencia positiva entre el número de nacidos vivos y el de fallecidos es para España del 10,7 por 1.000 habitantes, y para la Región estudiada se eleva al 11,8; sólo la Provincia de Madrid les supera con el 14,9. Las restantes Provincias de la Región presentan crecimientos vegetativos mucho más modestos, desde el 9,6 de Guadalajara al 7,4 de Ciudad Real y el 5,9 de Toledo; las demás, con valores inferiores, hasta llegar a Soria, con tan sólo el 0,5 para el año 1973.

En cuanto a las migraciones interiores, todas las Provincias son de despoblación y emigración, salvo Madrid, con una fuerte inmi- 
gración. La emigración exterior continental, muy reducida, como la de Ultramar, procede básicamente de las Provincias de Madrid y Albacete.

La población económicamente activa para el conjunto de la $\mathrm{Re}$ gión da un 34,4 por 100 del total, que se eleva al 56,6 para varones y alcanza al 13,5 para mujeres; y por sectores de actividad económica presenta el 16,4 en el primario, el 23,6 en el secundario y el 58 en el terciario. El detalle por Provincias es como sigue, asimismo expresado en porcentajes:

\begin{tabular}{|c|c|c|c|}
\hline & Totai & Varones & Miujeres \\
\hline 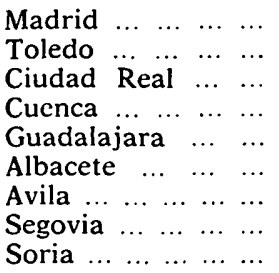 & $\begin{array}{l}35,8 \\
32,3 \\
30,6 \\
32,0 \\
33,2 \\
31,0 \\
34,3 \\
33,2 \\
33,4\end{array}$ & $\begin{array}{l}56,3 \\
58,0 \\
56,4 \\
57,0 \\
58,0 \\
55,4 \\
59,4 \\
55,7 \\
56,6\end{array}$ & $\begin{array}{r}16,9 \\
7,2 \\
6,2 \\
7,5 \\
7,8 \\
7,0 \\
9,3 \\
10,7 \\
10,6\end{array}$ \\
\hline & Primario & Secundario & Terciario \\
\hline 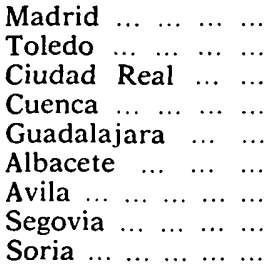 & $\begin{array}{r}2,2 \\
41,1 \\
39,4 \\
52,6 \\
42,2 \\
41,5 \\
50,5 \\
41,6 \\
43,9\end{array}$ & $\begin{array}{r}28,0 \\
18,3 \\
17,3 \\
9,2 \\
15,8 \\
16,7 \\
7,9 \\
15,2 \\
14,8\end{array}$ & $\begin{array}{l}67,5 \\
39,4 \\
41,5 \\
38,0 \\
41,5 \\
38,2 \\
40,5 \\
42,3 \\
40,5\end{array}$ \\
\hline
\end{tabular}

La población activa femenina destaca en la Provincia de Madrid, donde trabajan más de la sexta parte de las mujeres; pero es más reducida en las demás Provincias, si bien se apunta una situación más favorable en las tres Provincias septentrionales de la Región.

Por sectores de la actividad económica, la distribución transcrita, en la que no se ha consignado el "no consta», que completa los porcentajes respectivos, destaca, en primer lugar, el escaso porcentaje dedicado en la Provincia de Madrid a actividades del sector primario, que contrasta con los elevados que corresponden a las demás Provincias, corroborando el carácter predominantemente agrícola de su economía. Como complemento de esta afirma- 
ción, se observa el claro predominio del sector de servicios en todas ellas y los bajos niveles de la actividad industrial en todas las Provincias, a excepción de la de Madrid.

\section{LA GEOECONOMIA}

\section{LA INFRAESTRUCTURA}

Aborda la monografía comentada, en primer término, la infraestructura de la Región, con principal referencia a las vías de transporte y a las fuentes y consumo de energía, y, en segundo lugar, estudia las actividades más directamente relacionadas con el aprovechamiento de la tierra: agricultura, ganadería, silvicultura y la explotación de minas y canteras.

En cuanto a las vias de transporte, la red de carreteras del Estado tiene en la Región una longitud de $16.512 \mathrm{~km}$., el 21 por 100 de la red nacional, y la de carreteras provinciales y caminos vecinales es, a su vez, de $10.284 \mathrm{~km}$., el 16,39 del total nacional, lo que evidencia que no está bien dotada de ambas clases de carreteras, a pesar de su situación central. Existen, sin embargo, 96,5 km. de autopistas, de los que 44,5 son de peaje, y $120 \mathrm{~km}$. de autovías de libre circulación. Lo propio ocurre con la red ferroviaria, de $2.550 \mathrm{~km}$., que supone $22,6 \mathrm{~m}$. de vía por $\mathrm{km}^{2}$, mientras que para el conjunto de España se dan $31,6 \mathrm{~m}$. de vía por $\mathrm{km}^{2}$, a pesar del sistema radial de nuestra red, con centro y punto de arranque en Madrid; existen, además, $58 \mathrm{~km}$. de ferrocarril metropolitano y suburbano en Madrid. Se dispone del aeropuerto de Barajas, el primero del país por el tráfico regular de viajeros. Atravesada la Región por el oleoducto Rota-Zaragoza, dispone de los ramales Puertollano-Almodóvar, Loeches-Villaverde y Loeches-Barajas, con una longitud total de $59 \mathrm{~km}$.

Las fuentes de energía de la Región están constituidas por las 292 centrales eléctricas, de ellas 28 térmicas y una nuclear, la de Zorita, con una potencia instalada de $1.770 .519 \mathrm{kw}$., que supone el 8 por 100 de la nacional, y una producción de $3.934 .241 \mathrm{Mk} / \mathrm{h}$., que representa el 6 por 100 del conjunto nacional y que no llega a cubrir la mitad del consumo de la Región; la fábrica de gas ciudad de Madrid, con una producción de 279 millones de $\mathrm{m}^{3}$; y las minas y factoría de Puertollano, que produce 379.000 toneladas de carbones, 
que cubren el 50 por 100 de las necesidades, 58.915 toneladas de butano y 19.078 de propano, 228 millones de $\mathrm{m}^{3}$ de gases de refinería y 318.428 toneladas de gases licuados, insuficientes para cubrir las necesidades de consumo de la Región, que resulta también deficitaria en energía.

\section{LA AGRICULTURA}

Con referencia a la agricultura, la superficie labrada es de 5,4 millones de hectáreas, de ellas 5,2 en secano y el resto en regadío, y representan el 25,7 por 100 de la superficie labrada peninsular, el 27,6 de la de secano y el 10,7 de la de regadío. La superficie sembrada y plantada fue, en la campaña 1971-72, de 3,9 millones de hectáreas, el 22 por 100 de la nacional. Los cultivos principales son: los cereales, con 31,4 millones de quintales, el 23 por 100 de la producción nacional; las leguminosas, 1,3 millones de quintales, .que equivale al 23 por 100 de la producción nacional; los cultivos industriales, con el 31 por 100 , y el viñedo, con el 44,5 por 100 del total nacional; alrededor del 10 por 100 del total nacional suponen, separadamente, los tubérculos, remolacha, plantas oleaginosas, hortalizas y cultivos forrajeros. El parque regional de maquinaria agricola comprende, respecto al nacional, el 23 por 100 de los motores, el 21 de los tractores, el 25 de sembradoras y el 27 de cosechadoras, y resulta moderadamente deficitario, teniendo en cuenta el porcentaje de la superficie labrada. El riego por aspersión, a 73.500 hectáreas.

\section{LA SILVICULTURA}

En orden a la silvicultura tengamos presente que la superficie forestal es de 5,2 millones de hectáreas, el 46,3 por 100 de la superficie de la Región y la quinta parte de la superficie forestal española. El 65 por 100 de la superficie forestal pertenece a particulares, siguiéndole en importancia los montes de utilidad pública no consorciados, los consorciados, los de Corporaciones locales y, finalmente, los del Estado. El 42 por 100 de la superficie forestal está cubierta con especies arbóreas, de las que el 54 por 100 son coniferas. El 85 por 100 de los montes poblados con especies no 
arbóreas son matorrales, y el resto, praderas naturales o espartales. Por la importancia de los montes poblados con especies arbóreas destacan las Provincias de Albacete, Cuenca, Segovia y Soria en coníferas, y Ciudad Real, Toledo y Guadalajara en frondosas.

\section{LA GANADERÍA}

La cabaña regional ganadera se elevaba en 1973 a 6,6 millones de cabezas, equivalente al 19,16 por 100 de la nacional, de las que 4,6 corresponden al ganado ovino, que representa el 26,7 del total de la Nación, siguiéndole en importancia las especies porcina, bovina y caprina. El ganado ovino se da en toda la Región, pero especialmente en las Provincias de Cuenca, Ciudad Real, Toledo y Soria. En cuanto a las producciones ganaderas, destaca la de carne, que supone el 16 por 100 de la nacional; de leche, con el 11; de huevos, con el 15; de lana, con el 20, y la de miel, que representa el 17. Madrid es la principal Provincia productora de carne y leche.

\section{LA EXPLOTACIÓN DE MINAS Y CANTERAS}

La producción minera de la Región es poco importante en relación con la nacional, salvo con referencia a algunos minerales, como el mercurio de Almadén y el caolín. Por el contrario, la explotación de canteras alcanza niveles importantes en relación con el conjunto nacional, especialmente en orden a la extracción de arcilla, arena y arenisca, caliza, granito y yeso.

\section{LA INDUSTRIA}

En la monografía que comentamos se utiliza el Censo de contribuyentes con licencia fiscal, ya que es el único que proporciona los datos necesarios para el conocimiento de la composición por actividades de la industria a nivel municipal, aunque es bien sabido que el concepto «contribuyente» no coincide con el de "establecimiento", ya que éstos se contabilizan más de una vez como contribuyentes, atendiendo a las licencias fiscales necesarias. La distribución de contribuyentes por Provincias es la siguiente: 
ESTADISTICA

\begin{tabular}{|c|c|c|c|}
\hline & Provincias & Capitales & $\begin{array}{l}\text { Provincias } \\
\text { sin capitales }\end{array}$ \\
\hline Madrid & 40.417 & 31.224 & 9.193 \\
\hline $\begin{array}{lllll}\text { Toledo } & \ldots & \ldots & \ldots & \ldots\end{array}$ & 8.464 & 649 & 7.815 \\
\hline Ciudad Real $\ldots \quad \ldots$ & 6.504 & 368 & 6.136 \\
\hline $\begin{array}{lllll}\text { Cuenca } & \ldots & \ldots & \ldots & \ldots\end{array}$ & 3.942 & 492 & 3.450 \\
\hline $\begin{array}{ccc}\text { Guadalajara } & \ldots & \ldots\end{array}$ & 2.197 & 497 & 1.700 \\
\hline $\begin{array}{llll}\text { Albacete } & \ldots & \ldots & \ldots\end{array}$ & 5.211 & 1.449 & 3.762 \\
\hline $\begin{array}{lllllll}\text { Avila } & \ldots & \ldots & \ldots & \ldots & \ldots\end{array}$ & 2.190 & 379 & 1.811 \\
\hline $\begin{array}{lllll}\text { Segovia } & \ldots & \ldots & \ldots & \ldots\end{array}$ & 2.402 & 575 & 1.827 \\
\hline $\begin{array}{lllllll}\text { Soria } & \ldots & \ldots & \ldots & \ldots & \ldots\end{array}$ & 1.715 & 456 & 1.259 \\
\hline
\end{tabular}

En el conjunto de la Región existen 73.042 contribuyentes, distribuidos en dos partes casi iguales en las capitales y en las Provincias sin capitales, con 36.089 y 36.953 respectivamente. Si separamos la Provincia de Madrid, que concentra en la capital más de las tres cuartas partes de los contribuyentes, la distribución del resto de contribuyentes entre las Provincias resulta muy desigual, pues en las restantes capitales figuran tan sólo 4.865 contribuyentes frente a 27.760 en las Provincias sin capital.

Por ramas de actividad, los contribuyentes se distribuyen de esta manera:

\begin{tabular}{|c|c|c|c|}
\hline & Provincias & Capitales & $\begin{array}{l}\text { Provincias } \\
\text { sin capitales }\end{array}$ \\
\hline Alimentación y bebidas ... ... & 15.907 & 2.611 & 13.296 \\
\hline $\begin{array}{lllll}\text { Textil y confección } & \ldots & \ldots & \ldots\end{array}$ & 4.175 & 2.946 & 1.229 \\
\hline Madera y corcho $\ldots \ldots \ldots \ldots$ & 7.570 & 3.803 & $3.767^{\circ}$ \\
\hline Papel y artes gráficas ........ & 3.311 & 2.853 & 458 \\
\hline Piel y calzado $\ldots \ldots \ldots \ldots \ldots$ & 2.558 & 1.864 & 694 \\
\hline $\begin{array}{lllllll}\text { Químicas } & \ldots & \ldots & \ldots & \ldots & \ldots & \ldots\end{array}$ & 1.822 & 1.019 & 803 \\
\hline Construcción, vidrio, cerám. & 11.110 & 5.614 & 5.496 \\
\hline Metal $\ldots \ldots \ldots \ldots$ & 22.129 & 13.071 & 9.058 \\
\hline Energía, gas y agua $\ldots \ldots \ldots$ & 1.483 & 334 & 1.149 \\
\hline Actividades diversas $\ldots \ldots \ldots$ & 2.977 & 1.974 & 1.003 \\
\hline
\end{tabular}

Se advierte un predominio de industrias de la alimentación, bebidas y de energía y agua en las Provincias sin capital; de las industrias textil y de confección, papel y artes gráficas, piel y calzado y químicas en las capitales, y una importancia similar en unas y otras con respecto a las industrias de madera y corcho, construcción, vidrio y cerámica y metal.

Se registra un marcado predominio de la Provincia de Madrid en todas las ramas de actividad industrial, como es lógico, ya que 
es la más industrializada. Junto a ella tienen relativa importancia Toledo, Ciudad Real, Albacete y Cuenca, en alimentación y bebidas; Toledo, en construcción, vidrio y cerámica, y Toledo, Albacete y Ciudad Real, en el metal.

Los establecimientos industriales inscritos en las Delegaciones Provinciales de Industria son, en el conjunto de la Región, para el año 1973, 43.861, con una potencia instalada de 1,5 millones de kw. y 603.313 personas empleadas.

Según su tamaño y personal empleado, se distribuyen los establecimientos en esta forma:

\begin{tabular}{|c|c|c|}
\hline & $\begin{array}{c}\text { Número de } \\
\text { establecimiéntos }\end{array}$ & $\begin{array}{r}\text { Personal } \\
\text { empleado }\end{array}$ \\
\hline De menos de 6 obreros $\ldots \ldots$ & 33.229 & 79.645 \\
\hline 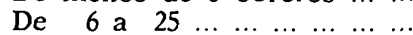 & 7.840 & 96.600 \\
\hline 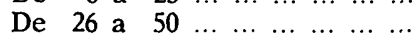 & 1.489 & 52.576 \\
\hline $\operatorname{De} 51$ a $100 \ldots \ldots \ldots \ldots$ & 640 & 45.073 \\
\hline $\begin{array}{llllllllll} & \\
\text { De } & 101 & \text { a } & 500 & \ldots & \ldots & \ldots & \ldots & \ldots & \ldots\end{array}$ & 551 & 113.977 \\
\hline De más de $500 \begin{array}{lllll} & \ldots & \ldots & \ldots & \ldots\end{array}$ & 112 & 215.442 \\
\hline
\end{tabular}

El 54 por 100 de la población activa industrial se concentra en establecimientos con más de 100 obreros. Estos grandes establecimientos, en un 85 por 100 se hallan ubicados en la Provincia de Madrid.

La distribución de los establecimientos y su mano de obra correspondiente, por Provincias, es ésta:

\begin{tabular}{|c|c|c|}
\hline & $\begin{array}{l}\text { Número de } \\
\text { establecimientos }\end{array}$ & $\begin{array}{l}\text { Personal } \\
\text { empleado }\end{array}$ \\
\hline Madrid & 24.922 & 509.092 \\
\hline $\begin{array}{lllllll}\text { Toledo } & \ldots & \ldots & \ldots & \ldots & \ldots & \ldots\end{array}$ & 3.445 & 16.417 \\
\hline Ciudad Real $\ldots \ldots \ldots c c$ & 4.169 & 23.293 \\
\hline $\begin{array}{lllllll}\text { Cuenca } & \ldots & \ldots & \ldots & \ldots & \ldots\end{array}$ & 2.284 & 7.562 \\
\hline Guadalajara $\quad . . \ldots \ldots \ldots$ & 1.313 & 9.820 \\
\hline 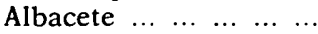 & 3.142 & 17.863 \\
\hline $\begin{array}{lllllll}\text { Avila } & \ldots & \ldots & \ldots & \ldots & \ldots & \ldots\end{array}$ & 1.413 & 3.509 \\
\hline $\begin{array}{cccccc}\text { Segovia } & \ldots & \ldots & \ldots & \ldots & \ldots\end{array}$ & 1.982 & 9.302 \\
\hline $\begin{array}{ccccccc}\text { Soria } & \ldots & \ldots & \ldots & \ldots & \ldots & \ldots\end{array}$ & 1.191 & 6.455 \\
\hline
\end{tabular}

Queda patente una vez más el alto nivel de industrialización de la Provincia de Madrid, que destaca sobre todas las demás, entre las que, atendiendo al personal empleado, sobresalen, a segundo nivel, Ciudad Real, Albacete y Toledo. 
Atendiendo al valor de la producción, por ramas de actividad, en 1972, en Región estudiada, la producción de cada rama supone del 12 al 20 por 100 de la producción nacional respectiva; la más baja corresponde a las industrias de la alimentación, y la más elevada, a construcción, vidrio y cerámica.

De 1969 a 1973, los números índices de producción industrial de la Provincia de Madrid denotan un incremento general y por ramas, a excepción de las de textiles y confección y cuero y calzado.

Finalmente se da amplia información del desarrollo e industrias que comprenden cada uno de los polígonos industriales de la Región: Arroyomolinos, en la Provincia de Madrid; Toledo industrial; los de Alcázar de San Juan y Manzanares, en Ciudad Real; Los Palancares, en Cuenca; El Balconcillo, Henares y Miralcampo, en Guadalajara; Campollano, en Albacete; Las Hervencias, en Avila, y El Cerro, en Segovia.

\section{EL COMERCIO}

El Censo de contribuyentes para el comercio en general, mayorista y minorista da para la Región 127.306 "puntos de venta» en 1973 , de los cuales 15.688 corresponden al comercio mayorista y 111.618 al minorista. Por Provincias, el detalle es el siguiente:

\begin{tabular}{|c|c|c|c|}
\hline & $\begin{array}{l}\text { Comercio } \\
\text { en generai }\end{array}$ & Mayorista & Minorista \\
\hline $\begin{array}{lllll}\text { Madrid } & \ldots & \ldots & \ldots & \ldots\end{array}$ & 86.054 & 11.701 & 74.353 \\
\hline $\begin{array}{llllll}\text { Toledo } & \ldots & \ldots & \ldots & \ldots\end{array}$ & 9.465 & 804 & 8.661 \\
\hline Ciudad Real ... ... & 8.785 & 641 & 8.144 \\
\hline $\begin{array}{llllll}\text { Cuenca } & \ldots & \ldots & \ldots & \ldots\end{array}$ & 4.200 & 305 & 3.895 \\
\hline $\begin{array}{lll}\text { Guadalajara } & \ldots & \ldots\end{array}$ & 2.904 & 296 & 2.608 \\
\hline $\begin{array}{llll}\text { Albacete } & \ldots & \ldots & \ldots\end{array}$ & 6.357 & 780 & 5.577 \\
\hline $\begin{array}{lllllll}\text { Avila } & \ldots & \ldots & \ldots & \ldots & \ldots\end{array}$ & 3.757 & 392 & 3.365 \\
\hline $\begin{array}{lllll}\text { Segovia } & \ldots & \ldots & \ldots & \ldots\end{array}$ & 3.633 & 574 & 3.059 \\
\hline $\begin{array}{lllllll} & \text { Soria } & \ldots & \ldots & \ldots & \ldots & \ldots\end{array}$ & 2.151 & 195 & 1.956 \\
\hline
\end{tabular}

La Provincia de Madrid absorbe el 75 por 100 del comercio ma. yorista y el 67 por 100 del minorista; las demás Provincias quedan muy alejadas de aquélla en cuanto al comercio interior. Las capitales de Provincia disponen del 75 por 100 del comercio mayorista 
y del 71 del minorista. Tras Madrid se sitúan, en el comercio mayorista y minorista, las Provincias de Toledo, Albacete y Ciudad Real y las ciudades de Toledo, Albacete, Ciudad Real, Avila y Segovia. Por ramas de actividad destacan, por su mayor número, los establecimientos de alimentación y bebidas, metal, químicas, textil y confección, por este orden; seguidas, en menor número, de los relativos a construcción, vidrio y cerámica, papel y artes gráficas, piel y calzado, madera y corcho.

Es de notar el desarrollo en los últimos años de unas formas modernas de establecimientos comerciales, cuales son los autoservicios, superservicios y supermercados, de los que existían en la Región, en 1972, 1.000 establecimientos, 800 de ellos autoservicios.

Una forma peculiar del comercio, tradicional en España, es la celebración de ferias y mercados que se reseñan nominativamente en cada Provincia, aunque los mercados han perdido gran parte de su importancia de antaño.

Igualmente se exponen en la monografía datos referentes a otra forma peculiar del comercio, el de las ventas a plazos, muy generalizada en las ciudades, en especial en Madrid, con un importe de 15.000 millones de pesetas en el año 1972.

En orden al comercio exterior hay que tener en cuenta que hay despacho de Aduanas en el aeropuerto de Barajas y en el de Albacete para el tráfico áereo, y en las estaciones de las Peñuelas y de Príncipe Pío de Madrid para el de carretera y ferrocarril, si bien la mayor parte del comercio exterior se realiza a través de las aduanas terrestres fronterizas y marítimas. Se presentan, sin embargo, datos fragmentarios, expresivos del movimiento comercial en dichas aduanas de las operaciones de exportación intervenidas por las respectivas Cámaras de Comercio por medio de los certificados de origen. Por las aduanas de Madrid se exportaron mercancías por valor de 10.563 millones de pesetas en 1973 y se importaron por un importe de 61.702 millones.

\section{EL SECTOR DE SERVICIOS}

Para el conjunto de la Región existen 200.948 contribuyentes del sector, de los que 127.439 actúan en las capitales de Provincia y 73.509 en las Provincias sin capital. El detalle por Provincias es éste: 
ESTADISTICA

\begin{tabular}{|c|c|c|c|}
\hline & Provincias & $\begin{array}{c}\text { Capitales } \\
\text { de provincia }\end{array}$ & $\begin{array}{l}\text { Provincias } \\
\text { sin capital }\end{array}$ \\
\hline Madrid ... & 141.238 & 114.770 & 26.468 \\
\hline $\begin{array}{lllll}\text { Toledo } & \ldots & \ldots & \ldots & \ldots\end{array}$ & 13.575 & 1.436 & 12.139 \\
\hline Ciudad Real ... ... & 10.356 & 1.133 & 9.223 \\
\hline $\begin{array}{lllll}\text { Cuenca } & \ldots & \ldots & \ldots & \ldots\end{array}$ & 6.965 & 1.329 & 5.636 \\
\hline Guadalajara $\ldots \quad \ldots$ & 4.612 & 1.350 & 3.262 \\
\hline Albacete $\ldots \ldots \ldots$ & 8.731 & 3.287 & 5.444 \\
\hline Avila $\ldots \ldots \ldots \ldots$ & 5.910 & 1.180 & 4.730 \\
\hline $\begin{array}{lllll}\text { Segovia } & \ldots & \ldots & \ldots & \ldots\end{array}$ & 5.947 & 1.805 & 4.142 \\
\hline Soria $\ldots \ldots \ldots \ldots$ & 3.614 & 1.149 & 2.465 \\
\hline
\end{tabular}

Se observa, de una parte, el predominio de Madrid sobre el resto de las Provincias en el conjunto de la Región y la preponderancia de los centros de servicios en la Provincia sin capital, al menos numéricamente sobre las respectivas capitales. Se registra un incremento general del número de establecimientos de este sector.

Se lleva a cabo en esta publicación un análisis de cinco subsectores: Enseñanza, Servicios financieros, Hostelería y Turismo y Transportes.

En orden a la Enseñanza se registra la existencia de 3.336 establecimientos privados, de ellos 2.811 en las capitales - 2.620 en Madrid-y el resto en la Provincia sin capital, según el Censo de contribuyentes. $Y$ se transcriben las cifras de alumnos matriculados en el curso 1972-73 por niveles de enseñanza y clases de estudios, según el Instituto Nacional de Estadística, de cuyas cifras se deduce una vez más la preponderancia de Madrid, y especialmente su capital, en este servicio.

En relación con los Servicios financieros, los datos proceden del Consejo Superior Bancario y denotan la existencia de 1.455 establecimientos que realizan operaciones bancarias, 906 de ellos situados en las capitales y 549 en el resto de las Provincias que configuran la Región - de ellas 1.705 oficinas de los Bancos y de las Cajas de Ahorro-, y 394 que realizan operaciones de seguros y reaseguros.

En cuanto a Hosteleria y Turismo, según el Censo de contribuyentes, existen 2.650 establecimientos hoteleros de todas clases -2.228 de ellos en la Provincia de Madrid- y 23.842 establecimientos de servicios de alimentación. La capacidad de los primeros es de 56.539 plazas, de las que 43.725 corresponden a Madrid, si bien se refieren solamente a hoteles y pensiones. Funcionan en la Re- 
gión, además, 20 campings, con 7.320 plazas, y 19.459 campamentos turísticos y bungalows, con una capacidad de 93.552 plazas.

Estas cifras dan idea de la importancia turística de la zona, que tiene como centro Madrid y desde la que se desplazan anualmente a las restantes Provincias, en especial a Avila y Segovia, de una parte, y a Toledo, de otra, millones de turistas nacionales y extranjeros. En 1972 ocuparon los alojamientos hoteleros de la Región 1.708.663 viajeros, españoles y extranjeros residentes en España, que causaron 5.301.196 pernoctaciones, y 4.413.947 turistas extranjeros.

No puede silenciarse la existencia de 15 establecimientos hoteleros propiedad del Estado, con 4.117 plazas, con 17.825 estancias causadas por viajeros españoles y extranjeros y 460.000 cubiertos servidos.

Respecto a los medios de Transporte anotemos que el parque de vehículos automóviles -camiones, autobuses, turismos, motocicletas y tractores - a fines de 1973 era de 1.193.128 - de ellos 845.229 turismos-, que constituyen el 20 por 100 del parque nacional, que suponen 1.121 vehículos por 1.000 habitantes, o 148 habitantes por vehículo de turismo. El tráfico urbano se cifraba en $500 \mathrm{mi}$ llones de viajeros en autobuses y 530 millones en el ferrocarril metropolitano. El tráfico aéreo en el aeropuerto de Barajas está representado por 53.600 aeronaves entradas y salidas, con 3.700 .000 viajeros entrados y otros tantos salidos.

De esta forma se dispone de una información asaz interesante sobre esta Región Central, de la que hay que separar en todo caso a la Provincia y capital de Madrid, que de por sí constituyen ya una zona peculiar, con características cuantitativas para situarla a la cabeza de la Nación en muchos aspectos de la actividad nacional. El resto de la Región, formado por ocho Provincias de características más homogéneas, aunque con matices discriminatorios, pueden constituir una zona más equilibrada en los distintos aspectos demográficos y económicos. 
REVL-1975, núm. 188. BALLESTER ROS, IGNACIO. RASGOS DEMOGRAFICOS Y ECONOMICOS DE...

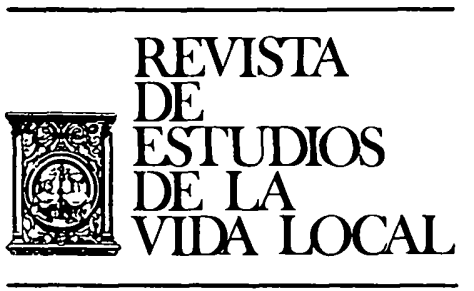

\section{JURISPRUDENCIA}


REVL-1975, núm. 188. BALLESTER ROS, IGNACIO. RASGOS DEMOGRAFICOS Y ECONOMICOS DE... 